PROFESIONALES Y HERRAMIENTAS PARA EL DESARROLLO LOCAL Y SUS SINERGIAS TERRITORIALES. EVALUACIÓN Y PROPUESTAS DE FUTURO IX Coloquio Nacional de Desarrollo Local del GTDL-AGE 

ANTONIO MARTÍNEZ PUCHE, XAVIER AMAT MONTESINOS, ISABEL SANCHO CARBONELL y DANIEL SANCHIZ CASTAÑO (EDS.)

\section{PROFESIONALES Y HERRAMIENTAS PARA EL DESARROLLO LOCAL Y SUS SINERGIAS TERRITORIALES. EVALUACIÓN Y PROPUESTAS DE FUTURO}

IX Coloquio Nacional de Desarrollo Local del GTDL-AGE

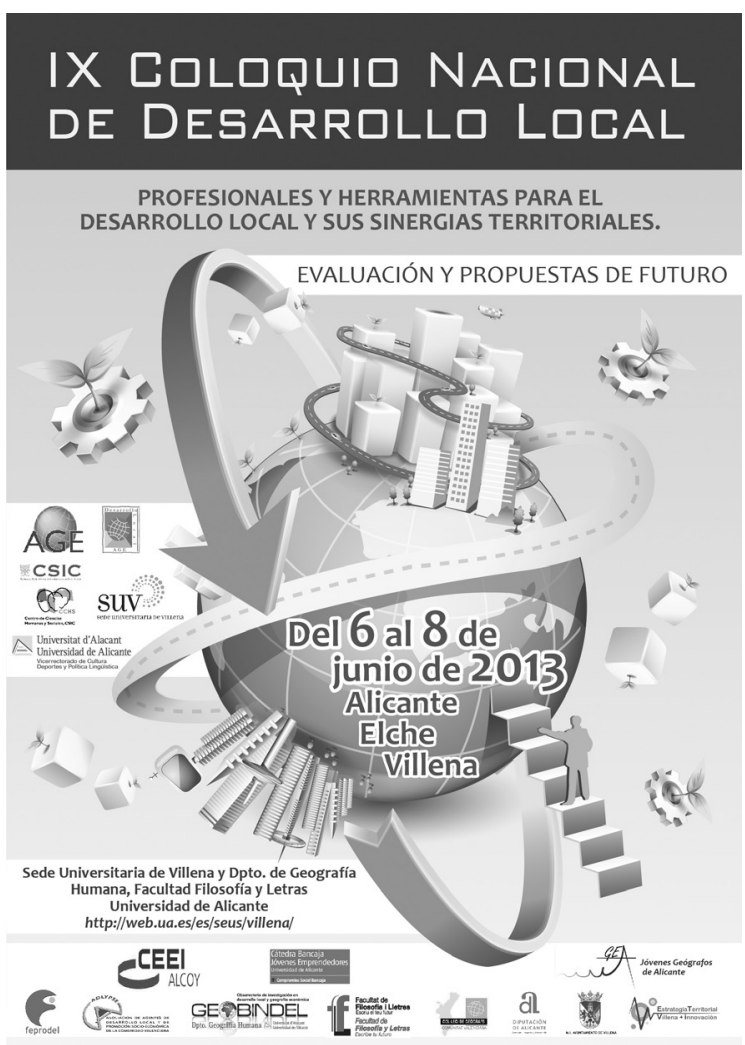


Este libro ha sido examinado y valorado por evaluadores ajenos a la Universidad de Alicante, con el fin de garantizar la calidad científica del mismo.

Publicacions de la Universitat d'Alacant

03690 Sant Vicent del Raspeig

Publicaciones@ua.es

http://publicaciones.ua.es

Telèfon: 965903480

(C) Antonio Martínez Puche, Xavier Amat Montesinos,

Isabel Sancho Carbonell y Daniel Sanchiz Castaño (eds.), 2016

(C) d'aquesta edició: Universitat d'Alacant

ISBN: 978-84-16724-00-0

Dipòsit legal: A 92-2016

Disseny de coberta: candela ink

Composició: Página Maestra (Miguel Ángel Sánchez Hernández)

Impressió i enquadernació: Guada Impresores

\section{unte \\ Unión de Editoriales
Universitarias Españolas \\ WWW.une.es
WWA}

Esta editorial es miembro de la UNE, cosa que garantiza la difusión y comercialización nacional y internacional de sus publicaciones.

Reservados todos los derechos. Cualquier forma de reproducción, distribución, comunicación pública o transformación de esta obra sólo puede ser realizada con la autorización de sus titulares, salvo excepción prevista por la ley. Diríjase a CEDRO (Centro Español de Derechos Repográficos, www.cedro.org) si necesita fotocopias o escanear algún fragmento de esta obra. 


\title{
CEEI ALCOY: 25 AÑOS APOYANDO LA INNOVACIÓN EN LAS EMPRESAS
}

\author{
Jordi Tormo Santonja \\ Centro Europeo de Empresas Innovadoras CEEI Alcoy \\ www.ceei-alcoy.com \\ jordi@ceei-alcoy.com
}

RESUMEN

El Centro Europeo de Empresas Innovadoras de Alcoi, es el $2^{\circ}$ que se creó en España. Ha estado más de dos décadas favoreciendo el emprendimiento y la innovación en las empresas de la provincia de Alicante. Además, ha apoya el fomentado el Desarrollo Local y la Innovación Territorial. La comunicación pretende visibilizar todas las acciones materializadas en el Territorio.

Palabras Clave: Desarrollo local, empresas y emprendimiento.

\section{AbSTRACT}

The European Center of Innovative Companies of Alcoi, is the $2^{\circ}$ that he was born in Spain. It has been more than twenty years favoring creation of companies and the innovation in the companies of the county of Alicante. Also, there is it supports the one fomented the Local Development and the Territorial Innovation. The text seeks to highlight all the actions materialized in the Territory.

Key words: Local Development, creation of companies.

\section{Estructura organizativa de LOS CEEI}

El CEEI Alcoy es una asociación privada sin ánimo de lucro creada en 1988 que tiene como objetivo promover la modernización del tejido industrial valenciano prestando su apoyo a todas aquellas iniciativas que supongan una innovación o diversificación empresarial. 
Es un instrumento para el impulso de la innovación y el desarrollo económico a través de la puesta en marcha de actividades y servicios para la creación de empresas innovadoras y el apoyo a las existentes en su proceso de innovación. El CEEI Alcoy promueve el emprendimiento, detecta proyectos innovadores, guía estratégicamente a las empresas desde su nacimiento hasta su puesta en marcha y lanzamiento al mercado, todo ello mediante la colaboración con organizaciones de soporte y/o apoyo empresarial.

Los Centros Europeos de Empresas Innovadoras (CEEI) o European Community Business and Innovation Centres (EC-BIC) son organismos de apoyo a las pymes y a los empresarios innovadores. Su creación fue promovida por el Instituto de la Mediana y Pequeña Industria Valenciana (IMPIVA), ahora Instituto Valenciano de Competitividad Empresarial (IVACE) organismo dependiente de la Consellería d'Economia, Indústria, Turisme i Ocupació, y está constituido por entidades, organizaciones y empresas de la provincia relacionados con los fines del Centro. Forma parte junto con los CEEI de la Comunidad Valenciana y los Institutos Tecnológicos de la Red de Servicios Avanzados promovida por el IVACE.

A nivel europeo, el CEEI-Alcoy es miembro de EBN (European Business and Innovation Centres Network). Esta asociación sin ánimo de lucro se encuentra localizada en Bruselas. EBN tiene como misión promover el desarrollo de los Centros Europeos de Empresas e Innovación dentro y fuera de la Unión Europea. EBN tiene como objetivo desarrollar vínculos entre los CEEI y otras instituciones de apoyo a las Pymes, mediante la asistencia técnica a miembros, creación de redes especializadas y desarrollo de proyectos europeos y la gestión de la marca BIC de la Unión Europea.

A nivel nacional pertenece a ANCES (Asociación Nacional de CEEI Españoles) constituida en 1994 con ámbito de actuación nacional. Es una asociación de carácter no lucrativo con personalidad jurídica propia que surgió con la idea de promover el desarrollo económico e industrial a través de los CEEI, aportando su experiencia sobre la creación de nuevas Pymes, basadas en elementos innovadores con potencial de desarrollo. Destaca el fomento y la difusión del papel de los CEEI como agentes claves entre la investigación, el desarrollo y la industria, el apoyo a organismos en la puesta en marcha de nuevos CEEI, y el establecimiento de lazos con los diferentes instrumentos ligados a la creación de empresas.

El CEEI Alcoy tiene como objeto apoyar la creación de nuevas empresas innovadoras e impulsar la mejora competitiva y el crecimiento de las existentes en el área que comprende la mitad norte de la provincia de Alicante y el Sur de la de Valencia. 


\section{El CEEI Alcoy: Misión y objetivos}

El CEEI Alcoy tiene como Misión ser el instrumento de soporte que sirva como catalizador del proceso de transformación del tejido empresarial, fomentando la innovación y la diversificación empresarial en el territorio, promoviendo empresas en nuevos sectores de actividad, ayudando a las empresas a diversificar y ser más competitivas y persiguiendo un desarrollo territorial que ofrezca un futuro mejor a sus ciudadanos. Y todo ello a través de la colaboración con otras entidades u organizaciones.

En síntesis, es una organización enfocada a apoyar la creación de nuevas empresas innovadoras, así como el impulso de la innovación en las existentes, para la mejora del tejido empresarial de su territorio de influencia. Para ello, pone en marcha y ofrece un sistema completo e integrado de actividades y servicios para los emprendedores y pymes de nueva creación con las siguientes líneas de actuación:

- Impulsar el espíritu emprendedor y empresarial.

- Apoyar a los emprendedores/innovadores en el proceso de creación de empresas.

- Ayudar en el proceso de consolidación y crecimiento de las empresas innovadoras de nueva creación.

- Fomentar nuevas líneas de actividad en las empresas existentes.

- Cooperar con otras entidades en estas actuaciones.

\section{SERVICIOS A EMPRENDEDORES, PYMES E INSTITUCIONES Y ORGANISMOS}

El CEEI Alcoy ha dedicado un esfuerzo importante durante los últimos años a realizar actividades relacionadas con la creación y consolidación de empresas. Desde 1988 ha promovido la constitución de más de 600 empresas que han supuesto una inversión total cercana a los 30 millones de euros y ha asistido a más de 260 empresas en procesos de crecimiento.

En concreto, es necesario destacar la realización de una serie de actividades concretas dentro de las áreas de asistencia a emprendedores y a empresas. A continuación se desarrolla una breve descripción de las actuaciones realizadas durante los últimos años en apoyo a los emprendedores:

\subsection{Sesiones individualizadas de atención y orientación a emprendedores}

Estas sesiones tienen como objetivo valorar el alcance y las necesidades de los emprendedores que se dirigen al CEEI solicitando un servicio relacionado con la puesta en marcha de sus proyectos empresariales. Mediante las sesiones de atención y orientación a personas emprendedoras los técnicos del CEEI dan a conocer las fuentes de información disponibles, así como las herramientas puestas a disposición del emprendedor por el propio CEEI u otros organismos. 
El objetivo es facilitar la valoración de la idea empresarial aportada por el emprendedor a fin de ofrecerle un servicio a medida de sus necesidades.

\subsection{Servicio de apoyo al emprendedor}

Una vez analizadas las necesidades del emprendedor portador de una idea de negocio, se le presta un servicio de asistencia técnica o/y elaboración del plan de negocio. Estos servicios engloban desde la primera información sobre la puesta en marcha de una empresa y el análisis de viabilidad de la idea, hasta la asistencia para la elaboración del plan de empresa, la búsqueda de financiación y la orientación y apoyo para la constitución de la empresa.

\subsection{Centro de empresas}

El Centro de Empresas del CEEI Alcoy es un instrumento de apoyo a la creación y puesta en marcha de proyectos empresariales innovadores. El Centro pone a disposición del emprendedor unas instalaciones totalmente acondicionadas para la ubicación de las empresas. El valor añadido que proporciona el centro es el apoyo del CEEI a las empresas instaladas, tanto en la fase previa a su instalación como durante su permanencia en el centro: asesoramiento e información permanente. Como se ha indicado arriba, el CEEI Alcoy dispone de 15 módulos para la instalación de empresas de reciente creación y que cuentan con todos los servicios necesarios para fortalecer su crecimiento.

\subsection{Desarrollo de herramientas de apoyo al emprendimiento}

Los CEEI ponen a disposición del emprendedor las fuentes de información y apoyo para la puesta en marcha de la empresa a través del canal web Emprenemjunts:

- Plan de empresa on-line: Plataforma on-line para la elaboración del plan del empresa por el emprendedor (http://planempresa.redceei.com/).

- Manuales de gestión empresarial: 40 manuales, con el objetivo de facilitar, de forma sencilla y con el apoyo de ejemplos prácticos, los conocimientos básicos del management que todo el emprendedor y empresario necesita para gestionar y dirigir su empresa (www.emprenemjunts.es).

- Enlaces emprendedores: Portal de enlaces de interés para el emprendedor (www.enlacesemprendedores.com).

- Guías de actividades empresariales: Información básica para iniciar una actividad empresarial resumida en sencillos estudios de mercado clasificados por sectores (www.guiasceei.com).

- Memofichas: Portal estructurado en diferentes secciones, con más de 200 fichas que recopilan los principales trámites y requisitos necesarios para la constitución y gestión diaria de una empresa (www.fichasceei.com). 
3.5. Organización del día de la persona emprendedora de la comunidad valenciana

El Día de la Persona Emprendedora de la Comunidad Valenciana (http://diadelapersonaemprendedora.emprenemjunts.es) es una iniciativa que se enmarca en la iniciativa nacional "Emprendemos Juntos", impulsada por el Ministerio de Industria. Se trata de una "macrojornada" con una asistencia superior a los 7.500 emprendedores según datos del 2012 y que agrupa un variado conjunto de actividades prácticas en horario de mañana y tarde organizadas en base a los siguientes espacios y actividades: Networking, Lonja de intercooperación, Oportunidades de negocio, Cápsulas y debates, Zona Conecta Vip, Ciudad del emprendedor,, Stands, Test del emprendedor, Box del emprendedor y Zona Chill Out. Además, se entregaron los premios al emprendedor. El CEEI Alcoy participa en calidad de entidad organizadora.

\subsection{Jornadas de difusión e impulso al emprendimiento y la innovación}

El objetivo general de estas jornadas es sensibilizar sobre los valores del emprendimiento como instrumento para el aumento de la competitividad empresarial y la mejora del empleo, la renta y la calidad de vida de la sociedad, así como el fomento del espíritu empresarial entre los jóvenes, el mundo universitario y la sociedad en general. Los contenidos de estas jornadas abarcan desde el fomento del espíritu empresarial hasta la difusión de herramientas necesarias para la dirección de una empresa, pasando por conferencias con expertos de reconocido prestigio en el ámbito empresarial sobre emprendimiento, innovación y oportunidades de negocio.

\subsection{Emprende +. difusión del espíritu emprendedor en institutos de educación secundaria}

Esta actividad se centra en la promoción del espíritu emprendedor en el alumnado de los ciclos formativos, mediante la realización de una serie de talleres sobre Capacidad emprendedora, Generación de ideas, Identificación de tendencias del mercado y Plan de empresa.

En este marco se elabora el Estudio de Vocación Emprendedora, análisis cuyo objetivo es medir el espíritu emprendedor de los jóvenes, conocer su capacidad, su espíritu creativo y competitivo y su resistencia al riesgo, así como identificar el grado de conocimiento del mundo de la empresa que tienen los jóvenes objeto del estudio.

\subsection{Emprendeaventur@}

Es un programa de capacitación de emprendedores que tiene como objetivo contribuir a la formación de los emprendedores que quieran poner en marcha 
iniciativas empresariales, apoyar técnicamente las iniciativas mediante la elaboración de los planes de negocio y apoyarlos económicamente a partir de la dotación de premios económicos. Una vez realizado el proceso formativo tiene lugar la fase de concurso de ideas empresariales.

\subsection{Premios CEEI-IMPIVA}

Estos premios, cuyo objetivo es el reconocimiento público a la labor de los emprendedores y empresas de reciente creación del ámbito del CEEI, se convocan anualmente por el CEEI Alcoy e IMPIVA en dos categorías: Mejor proyecto empresarial y Mejor trayectoria empresarial.

\subsection{Foro de financiación a la innovación}

Este Foro está orientado a Emprendedores y PYMES innovadoras con necesidades de financiación para su proyecto empresarial, inversores privados, instituciones financieras, entidades públicas y asociaciones empresariales.

El objeto del foro es poner en contacto a emprendedores y empresas y a entidades financieras públicas y privadas dispuestas a invertir su capital. Para ello se realiza la presentación de proyectos y la presentación de distintas modalidades de financiación.

\subsection{Portal Emprenemjunts. Canal CEEI Alcoy}

Uno de los objetivos principales del centro ha sido conseguir que la web Emprenemjunts (www.emprenemjunts.es) sea una plataforma de referencia en el ámbito del emprendimiento y la innovación, mediante la elaboración de contenidos propios. La finalidad ha sido acercar y difundir a través de las plataformas web de los CEEI, el conocimiento y las herramientas de apoyo al emprendimiento y al crecimiento empresarial al mayor número posible de usuarios de Internet.

\subsection{Lonja de inversión}

La Lonja de Inversión (http://lonjadeinversion.emprenemjunts.es) es un espacio web espacio donde las ideas innovadoras y el capital puedan encontrarse con el fin de posibilitar el desarrollo proyectos de inversión rentables y de gran potencial.

Esta herramienta funciona como un intermediador entre los emprendedores con un proyecto empresarial y que están en proceso de búsqueda de financiación y una red de inversores privados de proximidad puesta en marcha por los cuatro CEEI de la Comunidad Valenciana con el objetivo de financiar proyectos de elevado potencial de crecimiento.

Además, a continuación se desarrolla una breve descripción de las actuaciones realizadas y de servicios que el CEEI Alcoy pone a disposición de las empresas de su ámbito de actuación: 


\subsection{Sesiones individualizadas de atención a empresas}

Mediante estas sesiones los técnicos de apoyo al crecimiento valoran el alcance y las necesidades de las empresas que se dirigen al CEEI solicitando un servicio relacionado con la mejora de la gestión y el crecimiento empresarial, a fin de ofrecerle un servicio a medida.

\subsection{Acelerador. Servicio de apoyo al crecimiento}

Una vez analizadas las necesidades de la empresa, se presta un servicio diseñado a medida de sus necesidades. La tutela y asistencia a empresas se realiza principalmente en los siguientes temas:

- Asistencia técnica especializada en todas las áreas de la empresa.

- Valoraciones de empresas.

- Búsqueda de financiación para la Innovación: Business Angel.

- Estudios de viabilidad económico-financiera.

- Desarrollo de memorias orientadas al apoyo empresarial público.

- Gestión de proyectos multisectoriales.

- Apoyo en la elaboración de sistemas de cooperación entre empresas.

- Apoyo a la internacionalización, apertura de nuevos mercados.

- Elaboración de planes de innovación.

- Gestión de proyectos innovadores para las empresas.

\subsection{Programa de desarrollo de Planes Estratégicos en empresas}

El servicio prestado a las empresas participantes tiene como objetivo principal desarrollar con éxito el Plan Estratégico de cada una de ellas. Para ello, se tutela a cada una de las empresas participantes en un proceso de reflexión en relación con las alternativas de diversificación que se presentan en el mercado para desarrollar el plan que marque las directrices de trabajo de la empresa, alineando sus recursos hacia sus objetivos estratégicos.

\subsection{Club de empresas}

El Club de Empresas integra a las empresas CEEI que voluntariamente forman parte del mismo y se benefician de sus servicios. Es el punto de encuentro de todas aquellas compañías que apuestan por la innovación. La misión del Club es apoyar, contribuir y garantizar la consolidación, crecimiento y competitividad de las empresas en el mercado mediante la prestación de servicios de calidad.

\subsection{Financiación de la innovación. Red de Business Angels CEEI Alcoy}

Supone una herramienta excelente de financiación privada para los emprendedores y empresas y ofrece a los inversores privados la oportunidad de formar parte de negocios con elevado potencial de crecimiento. A través de la red, el 
CEEI pone en contacto a los emprendedores y empresas que tienen necesidades de financiación con inversores privados que buscan alternativas de inversión para su capital.

Por último, se debe destacar que el CEEI cuenta con una amplia experiencia en la gestión de proyectos financiados por distintos organismos y convocatorias, entre otras las del MITYC e IVACE. A continuación se indican los proyectos desarrollados durante los últimos años al amparo de convocatorias de ayudas y subvenciones y que han estado dirigidos principalmente a emprendedores y empresas:

Proyecto "CEAE. Inversión emprendedora para el crecimiento" financiado por el MITYC en 2011.

Proyecto "Consolidación de la red de business angels CEEI Alcoy" financiado por el MITYC en 2011.

Proyecto "Prestación de servicios avanzados para emprendedores e inversores de la red de business angels CEEI Alcoy" financiado por el MITYC en 2010.

\section{Actividades e indicAdores del CEEI Alcoy en 2012}

34 jornadas empresas 666 asistentes

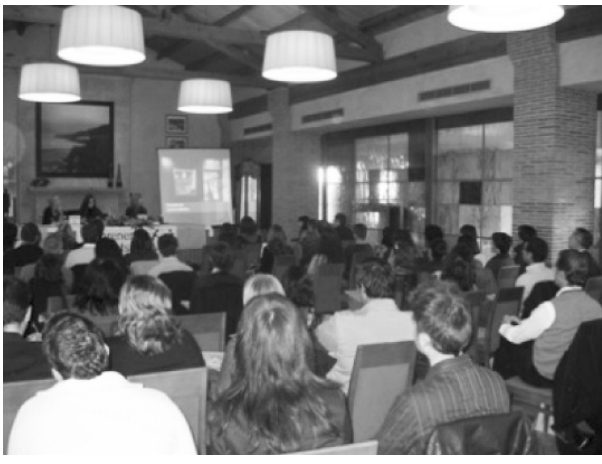

3 macrojornadas 4.451 asistentes

2 escuelas emprendedores

3 escuelas inversores

3 foros inversión

54 candidaturas a premios

171 asistencias individuales

173 consultas on line

15 sesiones E+ 298 asistentes

236 participantes concursos

153 usuarios herramientas on line

24 planes de empresa

25 planes crecimiento

12 empresas creadas

13 empresas instaladas CEM

2.116.250 € inversión inducida

484 empresas Club

18 inversores registrados

54 candidaturas premios

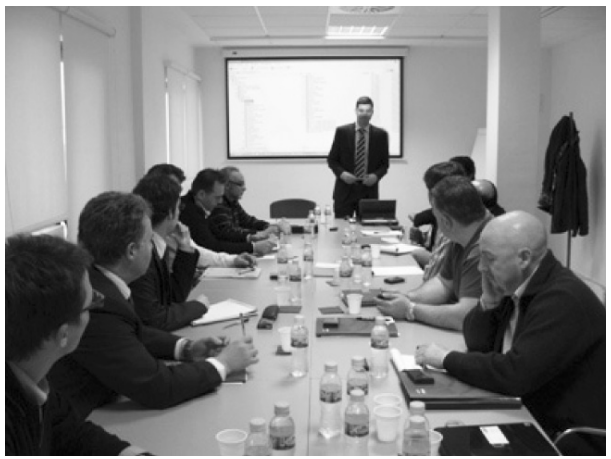




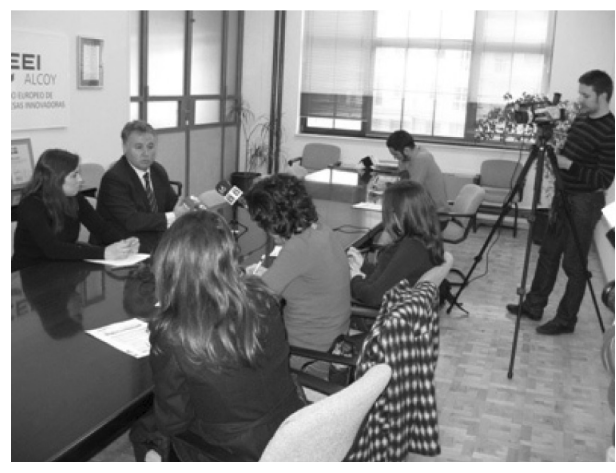

20 entidades colaboración

8 convenios de colaboración

25 jornadas en cooperación

413 asistentes

269.264 visitas web

124.055 visitantes

32.882 descargas documentos

515 documentos en web

2.932 usuarios registrados

2.380 seguidores redes sociales

14 ADL en web

68 boletines vía web

79 impactos en medios

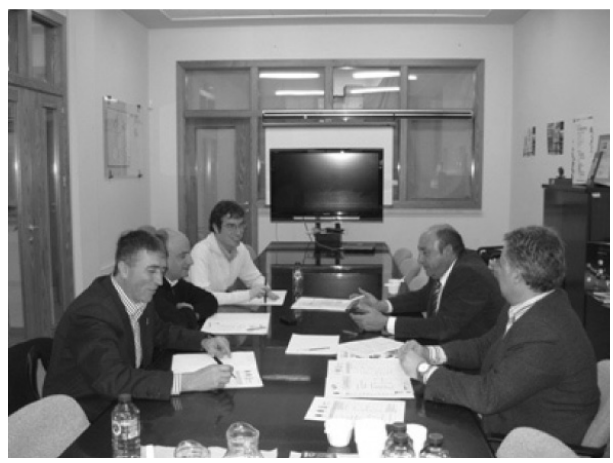

5. MÁs INFORMACIÓN

- Emprenemjunts: www.emprenemjunts.es

- CEEI Alcoy: www.ceei-alcoy.com

- Plan de empresa on-line: http://planempresa.redceei.com

- Enlaces emprendedores: www.enlacesemprendedores.com

- Guías de actividades empresariales: www.guiasceei.com

- Memofichas: www.fichasceei.com

- Lonja de Inversión: http://lonjadeinversion.emprenemjunts.es

- Día de la Persona Emprendedora: http://diadelapersonaemprendedora. emprenemjunts.es 